\title{
INFINITELY MANY SOLUTIONS FOR HARDY-HÉNON TYPE ELLIPTIC SYSTEM IN HYPERBOLIC SPACE
}

\author{
Haiyang He \\ Hunan Normal University, College of Mathematics and Computer Science \\ Changsha, Hunan 410081, P. R. China; hehy917@hotmail.com
}

\begin{abstract}
In this paper, we investigate the existence results for Hardy-Hénon type strongly indefinite elliptic system

$$
\left\{\begin{aligned}
-\Delta_{\mathbf{H}^{N}} u & =\left(d_{b}(x)\right)^{\alpha}|v|^{p-1} v \\
-\Delta_{\mathbf{H}^{N}} v & =\left(d_{b}(x)\right)^{\beta}|u|^{q-1} u
\end{aligned}\right.
$$

in the whole hyperbolic space $\mathbf{H}^{N}$, where $\alpha, \beta \in \mathbf{R}, N>4, d_{b}(x)=d_{\mathbf{H}^{N}}(b, x)$, and $b$ is a fixed point in hyperbolic space. We prove that there exist infinitely many nontrivial radial solutions for problem (0.1) under some suitable conditions.
\end{abstract}

\section{Introduction and main result}

In this article, we study the existence of nontrivial solutions for the following system with weights

$$
\left\{\begin{array}{l}
-\Delta_{\mathbf{H}^{N}} u=\left(d_{b}(x)\right)^{\alpha}|v|^{p-1} v \\
-\Delta_{\mathbf{H}^{N}} v=\left(d_{b}(x)\right)^{\beta}|u|^{q-1} u
\end{array}\right.
$$

on hyperbolic space $\mathbf{H}^{N}$, where $\alpha, \beta \in \mathbf{R}, \Delta_{\mathbf{H}^{N}}$ denotes the Laplace-Beltrami operator on $\mathbf{H}^{N}, N>4, d_{b}(x)=d_{\mathbf{H}^{N}}(b, x)$, and $b$ is a fixed point in hyperbolic space.

When posed in Euclidean space $\mathbf{R}^{N}$, problem (1.1) has two features. First it is the following problem

$$
\begin{cases}-\Delta u=|v|^{p-1} v, & \text { in } \Omega, \\ -\Delta v=|u|^{q-1} u, & \text { in } \Omega, \\ u=v=0, & \text { on } \partial \Omega .\end{cases}
$$

By a Pohozaev type identity, we know that the natural restriction on $p$ and $q$ is below a hyperbola, that is

$$
\frac{1}{p+1}+\frac{1}{q+1}>\frac{N-2}{N}
$$

see for instance [29]. Notice that the exponent $p$ or $q$ could be larger than $\frac{N+2}{N-2}$. Hence the usual Sobolev space $H_{0}^{1}(\Omega) \times H_{0}^{1}(\Omega)$ is not suitable to handle the problem. To study the problem (1.2) under the condition (1.3), a key observation was done by Hulshof and Van de Vorst [16], De Figueiredo and Felmer [9]. In order to solve this problem, the main idea is to destroy the symmetry between $u$ and $v$ by demanding more regularity of $u$ than that of $v$ if $p$ is large and $q$ is small, and vice versa. Thus

doi:10.5186/aasfm.2015.4056

2010 Mathematics Subject Classification: Primary 58J05, 35J60.

Key words: Hardy-Hénon type system, strongly indefinite, hyperbolic space. 
fractional Sobolev spaces are involved. A simple generalization of (1.2) to the case of systems is the following model

$$
\begin{cases}-\Delta u=|v|^{p-1} v, & \text { in } \mathbf{R}^{N}, \\ -\Delta v=|u|^{q-1} u, & \text { in } \mathbf{R}^{N} .\end{cases}
$$

Using a blow up technique, Qing [17] and Souto [25] had established the priori estimates for the solutions of problem (1.4). In [9], De Figueiredo and Felmer proved that if $0<p, q \leq \frac{N+2}{N-2}$, but not both are equal to $\frac{N+2}{N-2}$, then the only non-negative solution of (1.4) is the trivial one $u=0, v=0$.

Second, it is the following problem

$$
\begin{cases}-\Delta u=|x|^{\alpha}|v|^{p-1} v, & \text { in } \Omega, \\ -\Delta v=|x|^{\beta}|u|^{q-1} u, & \text { in } \Omega, \\ u=v=0, & \text { on } \partial \Omega .\end{cases}
$$

If $\alpha<0, \beta<0$, it is a Hardy type system. If $\alpha>0, \beta>0$, it is a Hénon type system. If $\alpha>0, \beta<0$, it is a Hardy-Hénon type system. We know that the hyperbola for the restriction of the exponents $p$ and $q$ will be affected by exponents $\alpha$ and $\beta$ of weighted functions $|x|^{\alpha}$ and $|x|^{\beta}$. Indeed, by Pohozaev type identities, we see that the natural restrictions on $p$ and $q$ become

$$
\frac{1}{p+1}\left(1+\frac{\alpha}{N}\right)+\frac{1}{q+1}\left(1+\frac{\beta}{N}\right)>\frac{N-2}{N},
$$

with $\alpha>-N, \beta>-N$. Such problem was studied in [10][18]. In [10], using a variational argument, De Figueiredo, Peral and Rossi proved that there exist infinitely many solutions of (1.5). Notice that if $p=q$, it is one equation case

$$
-\Delta u=|x|^{\alpha} u^{p} \text { in } \Omega, \quad u=0, \quad x \in \partial \Omega,
$$

where $\Omega$ is a bounded domain in $\mathbf{R}^{N}$. This Sobolev exponent is given by the corresponding Caffarelli-Kohn-Nirenberg estimate in [6]. For Hardy equation, critical and subcritical problems were considered, for instant, in [1, 7, 13]. For Hénon equation and its properties we refer to $[2,21,22,23,24]$ and the references therein. Among them, we must mention the famous paper [20]. It showed that $p(\alpha)=\frac{2(N+\alpha)}{N-2}$ is the critical exponents for the embedding of radially symmetric Sobolev spaces into $L^{p}$ spaces. In general domains, one has to restrict that the exponent $p+1$ is less than $\frac{2 N}{N-2}$.

It is also interest in studying problem (1.1) with respect to different ambient geometries in particular to see how curvature properties affect the existence and the nature of solutions. A recent paper by Mancini and Sandeep [19] had studied the existence / nonexistence and uniqueness of positive solution of the following elliptic equation

$$
-\Delta_{\mathbf{H}^{N}} u=|u|^{p-1} u+\lambda u
$$

in the subcritical case for every $\lambda<\left(\frac{N-1}{2}\right)^{2}$ and in critical exponent case for $\frac{N(N-1)}{4}<$ $\lambda \leq\left(\frac{N-1}{2}\right)^{2}$ with $N \geq 4$ on hyperbolic space $\mathbf{H}^{N}$. Moreover, they proved that if $\lambda=0$ and $1<p<\frac{N+2}{N-2}$, then problem (1.7) has a positive solution. This result is contrasted with the result in Euclidean space due to [12]. Afterward, Bhakta and Sandeep [4] had investigated the priori estimates, existence/nonexistence of radial sign changing 
solutions of problem (1.7). In [5], the classification of radial solutions of problem (1.7) was done by Bonforte etc. In [14], He applied the variational methods to study the following problem

$$
-\Delta_{\mathbf{H}^{N}} u=(d(x))^{\alpha}|u|^{p-1} u, \quad u \in H_{r}^{1}\left(\mathbf{H}^{N}\right)
$$

in hyperbolic space $\mathbf{H}^{N}$, and proved that there exists a positive solution of problem (1.8) provided that $p+1 \in\left(2, \frac{2 N+2 \alpha}{N-2}\right)$. For a bounded domain case, see [3, 26, 27]. In the case $\alpha=\beta=0$, He [15] considered the systems of semi-linear elliptic equations

$$
\left\{\begin{array}{l}
-\Delta_{\mathbf{H}^{N}} u=|v|^{p-1} v, \\
-\Delta_{\mathbf{H}^{N}} v=|u|^{q-1} u,
\end{array}\right.
$$

in the whole hyperbolic space $\mathbf{H}^{N}$. He established the decay estimates and the symmetry properties of positive solutions and proved that there is a positive solution pair $(u, v) \in H^{1}\left(\mathbf{H}^{N}\right) \times H^{1}\left(\mathbf{H}^{N}\right)$ of problem (1.9), moreover a ground state solution is obtained. Furthermore, He also proved that the above problem has a radial positive solution.

Our main concern in this paper is to look at the role played by the two weights when dealing with the existence of solutions. We find the existence of a critical hyperbola, given by,

$$
\frac{N+\alpha}{p+1}+\frac{N+\beta}{q+1}=N-2
$$

Below this hyperbola we prove that there exist infinitely nontrivial solutions for problem (1.1).

The difficulties in treating system (1.1) originate in at least three facts. Firstly, the weight function $d_{b}(x)$ depends on the Riemannian distance $r$ from a pole $b$. Secondly, due to the type of growth of the nonlinear term, we can not work with the usual space $H^{1}\left(\mathbf{H}^{N}\right)$ and then we need inhomogeneous Sobolev space. Thirdly, although problem (1.1) has a variational structure, the functional associated to it is strongly indefinite. Below we will show that we can overcome these difficulties by restricting to the radial situation.

It is well known that if $(u, v) \in H^{1}\left(\mathbf{R}^{N}\right) \times H^{1}\left(\mathbf{R}^{N}\right)$ is a solution of the following problem

$$
\left\{\begin{aligned}
-\Delta_{\mathbf{H}^{N}} u & =(d(x))^{\alpha}|v|^{p-1} v, \\
-\Delta_{\mathbf{H}^{N}} v & =(d(x))^{\beta}|u|^{q-1} u,
\end{aligned}\right.
$$

where $d(x)=\operatorname{dist}_{\mathbf{H}^{N}}(0, x)$, then $(\tilde{u}(x), \tilde{v}(x)=(u(\tau(x)), v(\tau(x))$ is the solution of $\operatorname{problem}(0.1)$, where $\tau$ is the hyperbolic translation such that $\tau(b)=0$. So, the main results in this paper are the following theorems under the assumption of $b=0$.

We first consider Hardy type systems, namely

$$
\left\{\begin{aligned}
-\Delta_{\mathbf{H}^{N}} u & =\frac{1}{(d(x))^{\alpha}}|v|^{p-1} v, \\
-\Delta_{\mathbf{H}^{N}} v & =\frac{1}{(d(x))^{\beta}}|u|^{q-1} u .
\end{aligned}\right.
$$

We have the following results.

Theorem 1.1. Let $0 \leq \alpha, \beta \leq N, \alpha+\beta<4, p, q>1$ satisfy

$$
\frac{1}{p+1}\left(1-\frac{\alpha}{N}\right)+\frac{1}{q+1}\left(1-\frac{\beta}{N}\right)>\frac{N-2}{N}
$$


and

$$
q+1<\frac{2(N-\beta)}{N-4}, \quad p+1<\frac{2(N-\alpha)}{N-4}, \quad \text { if } N>4
$$

then there exist infinitely many radial solutions and at least one positive radial solution of (1.11).

For Hénon type system

$$
\left\{\begin{aligned}
-\Delta_{\mathbf{H}^{N}} u & =(d(x))^{\alpha}|v|^{p-1} v \\
-\Delta_{\mathbf{H}^{N}} v & =(d(x))^{\beta}|u|^{q-1} u
\end{aligned}\right.
$$

we obtain

Theorem 1.2. Let $0 \leq \alpha, \beta, \frac{\alpha N}{N+\alpha}+\frac{\beta N}{N+\beta}<4, p, q>1$ satisfy

$$
\frac{1}{p+1}\left(1+\frac{\alpha}{N}\right)+\frac{1}{q+1}\left(1+\frac{\beta}{N}\right)>\frac{N-2}{N}
$$

and

$$
q+1<\frac{2(N+\beta)}{N-4}, \quad p+1<\frac{2(N+\alpha)}{N-4}, \quad \text { if } N>4
$$

then problem (1.12) possesses infinitely many radial solutions and at least one positive radial solution.

For Hardy-Hénon type system

$$
\left\{\begin{aligned}
-\Delta_{\mathbf{H}^{N}} u & =\frac{1}{(d(x))^{\alpha}}|v|^{p-1} v, \\
-\Delta_{\mathbf{H}^{N}} v & =(d(x))^{\beta}|u|^{q-1} u,
\end{aligned}\right.
$$

the existence results obtained are as follows.

Theorem 1.3. Let $0 \leq \alpha \leq N, 0 \leq \beta, p, q>1$ satisfy

$$
\frac{1}{p+1}\left(1-\frac{\alpha}{N}\right)+\frac{1}{q+1}\left(1+\frac{\beta}{N}\right)>\frac{N-2}{N}
$$

and

$$
q+1<\frac{2(N+\beta)}{N-4}, \quad p+1<\frac{2(N-\alpha)}{N-4}, \quad \text { if } N>4,
$$

then there are infinitely many radial solutions and at least one positive radial solution for (1.13).

Theorems 1.1-1.3 are proved by a linking theorem in a version that can by found in [8]. Due to the fact that $u$ and $v$ belong to different fractional Sobolev spaces so that the related functional may be well defined. So, we should establish the continuity and the compactness of the inclusions from fractional Sobolev spaces into $L_{\alpha}^{p}$ or $M_{\alpha}^{p}$ spaces.

This paper is organized as follows. In Section 2, we give some basic facts about hyperbolic space and present some compactness analysis. We obtain the embedding of fractional Sobolev spaces to weighted $L^{p}$ spaces and also establish the functional setting in which the problem will be posed in Section 3. In Section 4 we prove our main results. 


\section{Prelininaries}

The hyperbolic $N$-space $\mathbf{H}^{N}, N \geq 2$ is a complete simple connected Riemannian manifold having constant sectional curvature -1 , and for a given dimensional number, any two such spaces are isometric [30]. There are several models for $\mathbf{H}^{N}$, the most important being the half-space model, the ball model, and the hyperboloid or Lorentz model.

Let $\mathbf{B}^{N}=\left\{x \in \mathbf{R}^{N}:|x|<1\right\}$ denote the unit disc in $\mathbf{R}^{N}$. The space $\mathbf{H}^{N}$ endowed with the Riemannian metric $g$ given by $g_{i j}=(p(x))^{2} \delta_{i j}$ is called the ball model of the hyperbolic space, where $p(x)=\frac{2}{1-|x|^{2}}$. The hyperbolic gradient and the Laplace Beltrami operator are

$$
\begin{aligned}
\Delta_{\mathbf{H}^{N}} & \left.=\left(\frac{1-|x|^{2}}{2}\right)^{2} \Delta+(N-2) \frac{1-|x|^{2}}{2}\langle x, \nabla\rangle=(p(x))^{-N} \operatorname{div}\left((p(x))^{N-2} \nabla\right)\right), \\
\nabla_{\mathbf{H}^{N}} u & =\frac{\nabla u}{p(x)^{2}},
\end{aligned}
$$

where $\nabla$ and div denote the Euclidean gradient and divergence in $\mathbf{R}^{N}$, respectively. Let $H^{1}\left(\mathbf{H}^{N}\right)$ denote the Sobolev space on $\mathbf{H}^{N}$ with the above metric $g$, then we have $H^{1}\left(\mathbf{H}^{N}\right) \hookrightarrow L^{p}\left(\mathbf{H}^{N}\right)$ for $2 \leq p \leq \frac{2 N}{N-2}$ when $N \geq 3$, and $p \geq 2$ when $N=2$. From the Poincaré inequality

$$
\int_{\mathbf{H}^{N}}\left|\nabla_{\mathbf{H}^{N}} u\right|^{2} d V_{\mathbf{H}^{N}} \geq \frac{(N-1)^{2}}{4} \int_{\mathbf{H}^{N}}|u|^{2} d V_{\mathbf{H}^{N}}, \quad \forall u \in H^{1}\left(\mathbf{H}^{N}\right),
$$

we know that $\int_{\mathbf{H}^{N}}\left|\nabla_{\mathbf{H}^{N}} u\right|^{2} d V_{\mathbf{H}^{N}}$ is a norm, equivalent to the $H^{1}\left(\mathbf{H}^{N}\right)$.

The hyperbolic distance $d_{\mathbf{B}^{N}}(x, y)$ between $x, y \in \mathbf{B}^{N}$ in the Poincaré ball model is given by the formula

$$
d_{\mathbf{B}^{N}}(x, y)=\operatorname{Arccosh}\left(1+\frac{2|x-y|^{2}}{\left(1-|x|^{2}\right)\left(1-|y|^{2}\right)}\right) .
$$

From this we immediately obtain for $x \in \mathbf{B}^{N}$,

$$
d(x)=d_{\mathbf{B}^{N}}(0, x)=\ln \left(\frac{1+|x|}{1-|x|}\right) .
$$

Let $(u, v)$ be a symmetric solution of problem (1.1), and $u=u(|\xi|), v=$ $v(|\xi|),|\xi|<1$, then

$$
\left\{\begin{array}{l}
\left(\frac{1-|\xi|^{2}}{2}\right)^{2} \Delta u+(N-2) \frac{1-|\xi|^{2}}{2}\langle\xi, \nabla u\rangle+\left[\ln \left(\frac{1+|\xi|}{1-|\xi|}\right)\right]^{\alpha}|v|^{p-1} v=0 \\
\left(\frac{1-|\xi|^{2}}{2}\right)^{2} \Delta v+(N-2) \frac{1-|\xi|^{2}}{2}\langle\xi, \nabla v\rangle+\left[\ln \left(\frac{1+|\xi|}{1-|\xi|}\right)\right]^{\beta}|u|^{q-1} u=0 .
\end{array}\right.
$$

Setting $|\xi|=\tanh \frac{t}{2}, u(t)=u\left(\tanh \frac{t}{2}\right), v(t)=v\left(\tanh \frac{t}{2}\right), k(t)=(\sinh t)^{N-1}$, it is easy to see that

$$
\begin{aligned}
& \int_{\mathbf{H}^{N}}(d(x))^{\beta}|u|^{q+1} d V_{\mathbf{H}^{N}}=w_{N-1} \int_{0}^{\infty} t^{\beta} k(t)|u|^{q+1} d t \\
& \int_{\mathbf{H}^{N}}(d(x))^{\alpha}|v|^{p+1} d V_{\mathbf{H}^{N}}=w_{N-1} \int_{0}^{\infty} t^{\alpha} k(t)|v|^{p+1} d t
\end{aligned}
$$




$$
\begin{aligned}
& \int_{\mathbf{H}^{N}}\left|\nabla_{\mathbf{H}^{N}} u\right|^{2} d V_{\mathbf{H}^{N}}=w_{N-1} \int_{0}^{\infty} k(t)\left|u^{\prime}\right|^{2} d t, \\
& \int_{\mathbf{H}^{N}}\left|\nabla_{\mathbf{H}^{N}} v\right|^{2} d V_{\mathbf{H}^{N}}=w_{N-1} \int_{0}^{\infty} k(t)\left|v^{\prime}\right|^{2} d t,
\end{aligned}
$$

where $w_{N-1}$ denotes the surface area of $S^{N-1}$. In addition, we can rewrite (2.1) as

$$
\left\{\begin{array}{l}
u^{\prime \prime}+\frac{N-1}{\tanh t} u^{\prime}+t^{\alpha}|v|^{p-1} v=0 \\
v^{\prime \prime}+\frac{N-1}{\tanh t} v^{\prime}+t^{\beta}|u|^{q-1} u=0 \\
u^{\prime}(0)=v^{\prime}(0)=0
\end{array}\right.
$$

Let $H_{r}^{1}\left(\mathbf{H}^{N}\right)$ denote the subspace

$$
H_{r}^{1}\left(\mathbf{H}^{N}\right)=\left\{u \in H^{1}\left(\mathbf{H}^{N}\right): u \text { is radial }\right\} .
$$

It is well known that the critical exponent $p$ for the inclusion id: $H_{r}^{1}\left(\mathbf{H}^{N}\right) \hookrightarrow L^{p}$ is $p=\frac{2 N}{N-2}$ and the embedding id: $H_{r}^{1}\left(\mathbf{H}^{N}\right) \hookrightarrow L^{p}$ is compact for $2<p<\frac{2 N}{N-2}$.

For $0 \leq \alpha \leq p<N$, let

$$
L_{\alpha}^{p}=\left\{u: \int_{\mathbf{H}^{N}} \frac{|u|^{p}}{(d(x))^{\alpha}} d V_{\mathbf{H}^{N}}<+\infty\right\},
$$

then we have

Lemma 2.1. The embedding $H_{r}^{1}\left(\mathbf{H}^{N}\right) \hookrightarrow L_{\alpha}^{p}\left(\mathbf{H}^{N}\right)$ is continuous if $2 \leq p \leq$ $\frac{2(N-\alpha)}{N-2}$, and the embedding is compact if $2<p<\frac{2(N-\alpha)}{N-2}$.

Proof. Let $u \in H_{r}^{1}\left(\mathbf{H}^{N}\right)$, by [4][14], then we have

$$
|u(x)| \leq \frac{1}{\sqrt{\omega_{N-1}(N-2)}}\left(\frac{1-|x|^{2}}{2}\right)^{\frac{N-2}{2}} \frac{1}{|x|^{\frac{N-2}{2}}}\|u\|_{H_{r}^{1}\left(\mathbf{H}^{N}\right)},
$$

or

$$
|u(x)| \leq \frac{1}{\sqrt{\omega_{N-1}}}\left(\frac{1-|x|^{2}}{2}\right)^{\frac{N-1}{2}} \frac{1}{|x|^{\frac{N}{2}}}\|u\|_{H_{r}^{1}\left(\mathbf{H}^{N}\right)},
$$

where $\omega_{N-1}$ is the surface area of $S^{N-1}$.

Now, we claim that

$$
\int_{\mathbf{H}^{N}} \frac{|u|^{p}}{(d(x))^{\alpha}} d V_{\mathbf{H}^{N}} \leq C\|u\|_{H_{r}^{1}\left(\mathbf{H}^{N}\right)}^{p} \quad \text { for } p \in\left[2, \frac{2(N-\alpha)}{N-2}\right] .
$$

Indeed, if $N-\frac{N-2}{2} p-\alpha \geq 0$ and $\frac{N-1}{2} p-N+\alpha>-1$, we get

$$
\begin{aligned}
\int_{\mathbf{H}^{N}} \frac{|u|^{p}}{(d(x))^{\alpha}} d V_{\mathbf{H}^{N}} \leq & C\|u\|_{H_{r}^{1}\left(\mathbf{H}^{N}\right)}^{p}\left[\int_{0}^{\frac{1}{2}} \ln \left(\frac{1+r}{1-r}\right)^{-\alpha}\left(\frac{1-r^{2}}{2}\right)^{\left(\frac{N-2}{2} p-N\right)} r^{N-1-\frac{N-2}{2} p} d r\right. \\
& \left.+\int_{\frac{1}{2}}^{1} \log \left(\frac{1+r}{1-r}\right)^{-\alpha}\left(\frac{1-r^{2}}{2}\right)^{\left(\frac{N-1}{2} p-N\right)} r^{N-1-\frac{N}{2} p} d r\right]
\end{aligned}
$$




$$
\begin{aligned}
\leq & C\|u\|_{H_{r}^{1}\left(\mathbf{H}^{N}\right)}^{p}\left[\int_{0}^{\frac{1}{2}}\left(\frac{1-r^{2}}{2}\right)^{\left(\frac{N-2}{2} p-N+\alpha\right)} r^{N-1-\frac{N-2}{2} p-\alpha} d r\right. \\
& \left.+\int_{\frac{1}{2}}^{1}\left(\frac{1-r^{2}}{2}\right)^{\left(\frac{N-1}{2} p-N+\alpha\right)} r^{N-1-\frac{N}{2} p-\alpha} d r\right] \\
\leq & C\|u\|_{H_{r}^{1}\left(\mathbf{H}^{N}\right)}^{p} .
\end{aligned}
$$

This shows that the embedding $H_{r}^{1}\left(\mathbf{H}^{N}\right) \hookrightarrow L_{\alpha}^{p}\left(\mathbf{H}^{N}\right)$ is continuous if $2 \leq p \leq \frac{2(N-\alpha)}{N-2}$.

Now, we will show that it is compact. From [4], we know that $H_{r}^{1}\left(\mathbf{H}^{N}\right) \hookrightarrow L^{q}\left(\mathbf{H}^{N}\right)$ is compact for all $q \in\left(2, \frac{2 N}{N-2}\right)$. Then we fix $q \in\left(2, \frac{2(N-\alpha)}{N-2}\right)$. By the Hölder inequality for $a \in(0,1)$, we get

$$
\begin{aligned}
\int_{\mathbf{H}^{N}} \frac{|u(x)|^{p}}{(d(x))^{\alpha}} d V_{\mathbf{H}^{N}} & =\int_{\mathbf{H}^{N}} \frac{|u|^{p-q a}}{(d(x))^{\alpha}}|u|^{q a} d V_{\mathbf{H}^{N}} \\
& \left.\leq\left(\int_{\mathbf{H}^{N}}|u|^{q} d V_{\mathbf{H}^{N}}\right)^{a}\left[\int_{\mathbf{H}^{N}}(d(x))^{-\alpha}|u|^{p-q a}\right)^{\frac{1}{1-a}} d V_{\mathbf{H}^{N}}\right]^{1-a} .
\end{aligned}
$$

Now, we only need to check that

$$
p^{*}=\frac{p-q a}{1-a}<\frac{2\left(N-\frac{\alpha}{1-a}\right)}{N-2} .
$$

It is easy to check that (2.3) holds if and only if

$$
p(N-2)<2 N(1-a)+q a(N-2)-2 \alpha .
$$

Thus for a fixed $p<\frac{2(N-\alpha)}{N-2},(2.4)$ may easily be achieved by choosing $a$ sufficiently small. Hence, we have

$$
\|u\|_{L_{\alpha}^{p}\left(\mathbf{H}^{N}\right)} \leq\|u\|_{L^{q}\left(\mathbf{H}^{N}\right)}^{\frac{a}{p}}\|u\|_{H_{r}^{1}\left(\mathbf{H}^{N}\right)}^{\frac{1-a}{p}}
$$

where $a>0$ and is small. It is easy to see that this Lemma holds.

Let

$$
M_{\beta}^{q}=\left\{u: \int_{\mathbf{H}^{N}}(d(x))^{\beta}|u|^{q} d V_{\mathbf{H}^{N}}<+\infty\right\} .
$$

Lemma 2.2. The inclusion id: $H_{r}^{1}\left(\mathbf{H}^{N}\right) \hookrightarrow M_{\beta}^{q}$ is continuous if $2 \leq q \leq \frac{2(N+\beta)}{N-2}$ and is compact if $2<q<\frac{2(N+\beta)}{N-2}$.

Proof. See [14].

\section{The existence of radial solutions of (1.1)}

Now we define the inhomogeneous Sobolev space on $\mathbf{H}^{N}$. Let $L_{r}^{2}\left(\mathbf{H}^{N}\right)$ be the space of $L^{2}$-functions in $\mathbf{H}^{N}$ which are radially symmetric, and $T=-\Delta_{\mathbf{H}^{N}}$ with the domain $D(T)=H_{r}^{2}\left(\mathbf{H}^{N}\right)$ which is the space of radial symmetric functions that are in $L^{2}$ and have second derivatives in $L^{2}$. For $0 \leq s \leq 2$, the space $E^{s}$, which is the domain $D\left(T^{\frac{s}{2}}\right)$, is precisely the space obtained by interpolation between $H_{r}^{2}\left(\mathbf{H}^{N}\right)$ and $L_{r}^{2}\left(\mathbf{H}^{N}\right)$,

$$
\left[H_{r}^{2}\left(\mathbf{H}^{N}\right), L_{r}^{2}\left(\mathbf{H}^{N}\right)\right]_{1-\frac{s}{2}}
$$


In this case, the space $E^{s}$ is the usual Sobolev space $H_{r}^{s}\left(\mathbf{H}^{N}\right)$. So write $A=$ $\left(-\Delta_{\mathbf{H}^{N}}\right)^{\frac{1}{2}}$, we have for all $0 \leq s \leq 2$,

$$
D\left(A^{s}\right)=H_{r}^{s}\left(\mathbf{H}^{N}\right) .
$$

Moreover, we have $H_{r}^{s} \hookrightarrow H_{r}^{t}$ for $s>t$ (see [28]), and the Sobolev embedding theorem

$$
H_{r}^{s} \hookrightarrow L^{q}, \quad \text { if } 2 \leq q<\infty, \quad \text { and } \quad s=\frac{N}{2}-\frac{N}{q}
$$

holds.

Lemma 3.1. If $s>1$, then the space $H_{r}^{s}\left(\mathbf{H}^{N}\right)$ is continuous embedded in $L_{\alpha}^{\frac{2(N-\alpha)}{N-2 s}}\left(\mathbf{H}^{N}\right)$ and $M_{\beta}^{\frac{2(N+\beta)}{N-2 s}}$.

Proof. If $u \in H_{r}^{s}\left(\mathbf{H}^{N}\right)$ with $s>1$, then we have $u \in H_{r}^{1}\left(\mathbf{H}^{N}\right)$.

(i) We have

$$
\begin{aligned}
& \left(\int_{\mathbf{H}^{N}} \frac{|u|^{\frac{2(N-\alpha)}{N-2 s}}}{(d(x))^{\alpha}} d V_{\mathbf{H}^{N}}\right)^{\frac{N-2 s}{2(N-\alpha)}} \\
& =\left[\int_{B_{\frac{1}{2}}} \frac{|u|^{\frac{2(N-\alpha)}{N-2 s}}}{(d(x))^{\alpha}}\left(\frac{2}{1-|x|^{2}}\right)^{N} d x+\int_{B_{1} \backslash\left\{B_{\frac{1}{2}}\right\}} \frac{|u|^{\frac{2(N-\alpha)}{N-2 s}}}{(d(x))^{\alpha}}\left(\frac{2}{1-|x|^{2}}\right)^{N} d x\right]^{\frac{N-2 s}{2(N-\alpha)}} \\
& \leq C\|u\|_{H_{r}^{s}\left(\mathbf{H}^{N}\right)}+\left[\left(\int_{B_{1} \backslash\left\{B_{\frac{1}{2}}\right\}}|u|^{\frac{2 N}{N-2 s}}\left(\frac{2}{1-|x|^{2}}\right)^{N} d x\right)^{\frac{N-\alpha}{N}}\right. \\
& \left.\cdot\left(\int_{B_{1} \backslash\left\{B_{\frac{1}{2}}\right\}}(d(x))^{-N}\left(\frac{2}{1-|x|^{2}}\right)^{N} d x\right)\right]^{\frac{N-2 s}{2(N-\alpha)}} \\
& \leq C\|u\|_{H_{r}^{s}\left(\mathbf{H}^{N}\right)}+\left[\left(\int_{B_{1} \backslash\left\{B_{\frac{1}{2}}\right\}}|u|^{\frac{2 N}{N-2 s}}\left(\frac{2}{1-|x|^{2}}\right)^{N} d x\right)^{\frac{N-\alpha}{N}}\left(\int_{\frac{1}{2}}^{1} r^{-1} d x\right)\right]^{\frac{N-2 s}{2(N-\alpha)}} \\
& \leq C\|u\|_{H_{r}^{s}\left(\mathbf{H}^{N}\right)} .
\end{aligned}
$$

(ii) By [18], we have

$$
\begin{aligned}
& \left(\int_{\mathbf{H}^{N}}(d(x))^{\beta}|u|^{\frac{2(N+\beta)}{N-2 s}} d V_{\mathbf{H}^{N}}\right)^{\frac{N-2 s}{2(N+\beta)}} \\
& =\left[\int_{B_{\frac{1}{2}}}(d(x))^{\beta}|u|^{\frac{2(N+\beta)}{N-2 s}}\left(\frac{2}{1-|x|^{2}}\right)^{N} d x\right. \\
& \left.\quad+\int_{B_{1} \backslash\left\{B_{\frac{1}{2}}\right\}}(d(x))^{\beta}|u|^{\frac{2(N+\beta)}{N-2 s}}\left(\frac{2}{1-|x|^{2}}\right)^{N} d x\right]^{\frac{N-2 s}{2(N+\beta)}} \\
& \leq C\|u\|_{H_{r}^{s}\left(\mathbf{H}^{N}\right)}+\left[\int_{\frac{1}{2}}^{1}\left(\ln \frac{1+r}{1-r}\right)^{\beta \frac{2(N+\beta)}{N-2 s}}\left(\frac{1-r^{2}}{2}\right)^{\left(\frac{N-1}{2} \frac{2(N+\beta)}{N-2 s}-N\right)}\right. \\
& \left.\cdot r^{N-1-\frac{N}{2} \frac{2(N+\beta)}{N-2 s}} d r\right]^{\frac{N-2 s}{2(N+\beta)}}\|u\|_{H_{r}^{s}\left(\mathbf{H}^{N}\right)}
\end{aligned}
$$




$$
\begin{aligned}
\leq C\|u\|_{H_{r}^{s}\left(\mathbf{H}^{N}\right)}+ & {\left[\int_{\frac{1}{2}}^{1}\left(\frac{1-r^{2}}{2}\right){ }^{\left(\frac{N-1}{2} \frac{2(N+\beta)}{N-2 s}-N+\beta \frac{2(N+\beta)}{N-2 s}\right)}\right.} \\
& \left.\cdot r^{N-1-\frac{N}{2} \frac{2(N+\beta)}{N-2 s}-\beta \frac{2(N+\beta)}{N-2 s}} d r\right]^{\frac{N-2 s}{2(N+\beta)}}\|u\|_{H_{r}^{s}\left(\mathbf{H}^{N}\right)} \\
\leq C\|u\|_{H_{r}^{s}\left(\mathbf{H}^{N}\right)} . &
\end{aligned}
$$
[4].

Now, we have the following imbedding theorem. The case $s=1$ was proved by

Lemma 3.2. Let $s>0$. The restriction to $H_{r}^{s}\left(\mathbf{H}^{N}\right)$ of the Sobolev imbedding of $H^{s}\left(\mathbf{H}^{N}\right)$ into $L_{\alpha}^{p}\left(\mathbf{H}^{N}\right)$ is continuous if $2 \leq p \leq \frac{2(N-\alpha)}{N-2 s}$, and it is compact if $2<p<\frac{2(N-\alpha)}{N-2 s}$.

Proof. Case 1. $s>1$. By the definition of $H^{s}\left(\mathbf{H}^{N}\right)$, we have

$$
H_{r}^{s}\left(\mathbf{H}^{N}\right) \hookrightarrow H_{r}^{1}\left(\mathbf{H}^{N}\right) .
$$

So if $u \in H_{r}^{s}\left(\mathbf{H}^{N}\right)$, we have $u \in L_{\alpha}^{p}\left(\mathbf{H}^{N}\right), 2 \leq p \leq \frac{2(N-\alpha)}{N-2}$ and $u \in L^{q}\left(\mathbf{H}^{N}\right), 2 \leq q \leq$ $\frac{2 N}{N-2 s}$. For $\frac{2(N-\alpha)}{N-2}<p<\frac{2(N-\alpha)}{N-2 s}$, by Hölder inequality, we have

$$
\int_{\mathbf{H}^{N}} \frac{|u|^{p}}{(d(x))^{\alpha}} d V_{\mathbf{H}^{N}} \leq\left(\int_{\mathbf{H}^{N}} \frac{|u|^{\frac{2(N-\alpha)}{N-2}}}{(d(x))^{\alpha}}\right)^{b}\left(\int_{\mathbf{H}^{N}} \frac{|u|^{\frac{p-\frac{2(N-\alpha)}{N-2} b}{1-b}}}{d(x)^{\alpha}} d V_{\mathbf{H}^{N}}\right)^{1-b},
$$

where $0<b<1, \frac{1}{p}=\frac{\theta(N-2)}{2(N-\alpha)}+\frac{(1-\theta)(N-2 s)}{2(N-\alpha)}$. This shows that the map $i: H_{r}^{s}\left(\mathbf{H}^{N}\right) \rightarrow$ $L_{\alpha}^{p}\left(\mathbf{H}^{N}\right)$ is continuous for all $2 \leq p \leq \frac{2(N-\alpha)}{N-2 s}$.

Now, we will show that the map $i: H_{r}^{s}\left(\mathbf{H}^{N}\right) \rightarrow L_{\alpha}^{p}\left(\mathbf{H}^{N}\right)$ is compact for all $2<p<$ $\frac{2(N-\alpha)}{N-2 s}$. From [4], we have that $H_{r}^{1}\left(\mathbf{H}^{N}\right) \hookrightarrow L^{p}\left(\mathbf{H}^{N}\right), 2<p<\frac{2 N}{N-2}$ and $H_{r}^{1}\left(\mathbf{H}^{N}\right) \hookrightarrow$ $L_{\alpha}^{p}\left(\mathbf{H}^{N}\right), 2<p<\frac{2(N-\alpha)}{N-2}$ are compact. By the definition of $H^{s}\left(\mathbf{H}^{N}\right)$, we also have

$$
H_{r}^{s}\left(\mathbf{H}^{N}\right) \hookrightarrow H_{r}^{1}\left(\mathbf{H}^{N}\right) .
$$

Hence,

$$
H_{r}^{s}\left(\mathbf{H}^{N}\right) \hookrightarrow L^{p}\left(\mathbf{H}^{N}\right), \quad 2<p<\frac{2 N}{N-2}
$$

and

$$
H_{r}^{s}\left(\mathbf{H}^{N}\right) \hookrightarrow L_{\alpha}^{p}\left(\mathbf{H}^{N}\right), \quad 2<p<\frac{2(N-\alpha)}{N-2} \quad \text { are compact. }
$$

If $\frac{2(N-\alpha)}{N-2} \leq p<\frac{2(N-\alpha)}{N-2 s}$, we can deduce this lemma by using Sobolev inequalities and Hölder inequalities. Indeed, for $a \in(0,1)$ and $2<q<\frac{2(N-\alpha)}{N-2}$, we get

$$
\begin{aligned}
\int_{\mathbf{H}^{N}} \frac{|u|^{p}}{(d(x))^{\alpha}} d V_{\mathbf{H}^{N}} & =\int_{\mathbf{H}^{N}} \frac{|u|^{p-q a}|u|^{q a}}{(d(x))^{\alpha}} d V_{\mathbf{H}^{N}} \\
& \leq\left(\int_{\mathbf{H}^{N}} \frac{|u|^{q}}{(d(x))^{\alpha}} d V_{\mathbf{H}^{N}}\right)^{a}\left(\int_{\mathbf{H}^{N}} \frac{|u|^{\frac{p-q a}{1-a}}}{(d(x))^{\alpha}} d V_{\mathbf{H}^{N}}\right)^{1-a}
\end{aligned}
$$


Now, we only need to check that

$$
\frac{q-p a}{1-a} \leq \frac{2(N-\alpha)}{N-2 s}
$$

It is easy to check that (3.1) holds if and only if

$$
p(N-2 s) \leq 2(N-\alpha)(1-a)+q a(N-2 s)
$$

holds. Thus for a fixed $p<\frac{2(N-\alpha)}{N-2 s}$, (3.2) may easily be achieved by choosing $a$ sufficiently small.

Case 2. $0<s<1$. From [28], we have that

$$
\left[L^{2}\left(\mathbf{H}^{N}\right), H^{1}\left(\mathbf{H}^{N}\right)\right]_{s}=H^{s}\left(\mathbf{H}^{N}\right),
$$

and

Now, we claim that

$$
\left[L_{r}^{2}\left(\mathbf{H}^{N}\right), H_{r}^{1}\left(\mathbf{H}^{N}\right)\right]_{s}=H_{r}^{s}\left(\mathbf{H}^{N}\right)
$$

$$
\|u\|_{L_{\alpha}^{p}\left(\mathbf{H}^{N}\right)} \leq C\|u\|_{H^{s}\left(\mathbf{H}^{N}\right)} \quad \text { for } 2 \leq p \leq \frac{2(N-\alpha)}{N-2} .
$$

Indeed, suppose that $u \in L_{r}^{2}\left(\mathbf{H}^{N}\right) \cap H_{r}^{1}\left(\mathbf{H}^{N}\right)$, by Hölder inequality

$$
\begin{aligned}
\int_{\mathbf{H}^{N}} \frac{|u|^{p}}{(d(x))^{\alpha}} d V_{\mathbf{H}^{N}} & =\int_{\mathbf{H}^{N}} \frac{|u|^{2 \gamma_{1}+q\left(1-\gamma_{1}\right)}}{(d(x))^{\alpha}} d V_{\mathbf{H}^{N}} \\
& \leq\left(\int_{\mathbf{H}^{N}}|u|^{2} d V_{\mathbf{H}^{N}}\right)^{\gamma_{1}}\left(\int_{\mathbf{H}^{N}} \frac{|u|^{q}}{(d(x))^{\frac{\beta}{1-\gamma_{1}}}} d V_{\mathbf{H}^{N}}\right)^{1-\gamma_{1}}
\end{aligned}
$$

where $2 \gamma_{1}+q\left(1-\gamma_{1}\right)=p, 0<\gamma_{1}<1$. Hence

$$
\left(\int_{\mathbf{H}^{N}} \frac{|u|^{p}}{(d(x))^{\alpha}} d V_{\mathbf{H}^{N}}\right)^{\frac{1}{p}} \leq\|u\|_{L^{2}}^{\frac{2 \gamma_{1}}{p}}\left(\int_{\mathbf{H}^{N}} \frac{|u|^{q}}{(d(x))^{\frac{\alpha}{1-\gamma_{1}}}} d V_{\mathbf{H}^{N}}\right)^{\frac{1}{q} \frac{q\left(1-\gamma_{1}\right)}{p}} .
$$

Let $\theta_{1}=\frac{2 \gamma_{1}}{p}, \frac{q\left(1-\gamma_{1}\right)}{p}=1-\theta_{1}$. Then

$$
\left(\int_{\mathbf{H}^{N}} \frac{|u|^{p}}{(d(x))^{\alpha}} d V_{\mathbf{H}^{N}}\right)^{\frac{1}{p}} \leq\|u\|_{L^{2}}^{\theta_{1}}\left(\int_{\mathbf{H}^{N}} \frac{|u|^{q}}{(d(x))^{\frac{\alpha}{1-\gamma_{1}}}} d V_{\mathbf{H}^{N}}\right)^{\frac{1-\theta_{1}}{q}},
$$

and $\frac{1}{p}=\frac{\theta_{1}}{2}+\frac{1-\theta_{1}}{q}$. Let $\theta_{1}=1-s$. Then $q=\frac{2 p s}{2-p(1-s)}$ and $1-\gamma_{1}=1-\frac{p(1-s)}{2}$. By Lemma 2.1, we know that the critical exponent of

$$
\int_{\mathbf{H}^{N}} \frac{|u|^{q}}{(d(x))^{\frac{\alpha}{1-\gamma_{1}}}} d V_{\mathbf{H}^{N}}=\int_{\mathbf{H}^{N}} \frac{|u|^{q}}{(d(x))^{\frac{\alpha}{1-\frac{p(1-s)}{2}}}} d V_{\mathbf{H}^{N}}
$$

is $q^{*}(s)=\frac{2\left(N-\frac{2 \alpha}{2-p(1-s)}\right)}{N-2}$. Requiring $q \leq q^{*}(s)$, we obtain that

$$
p \leq \frac{2(N-\alpha)}{N-2 s} .
$$

Now, we want to prove that the embedding $H_{r}^{s}\left(\mathbf{H}^{N}\right) \hookrightarrow L_{\alpha}^{p}\left(\mathbf{H}^{N}\right)$ is compact if $2<p<\frac{2(N-\alpha)}{N-2 s}$. From Section 2, setting $|\xi|=\tanh \frac{t}{2}, u(t)=u\left(\tanh \frac{t}{2}\right), k(t)=$ $(\sinh t)^{N-1}$, we have

$$
\int_{\mathbf{H}^{N}} \frac{1}{(d(x))^{\alpha}}|u|^{p} d V_{\mathbf{H}^{N}}=w_{N-1} \int_{0}^{\infty} t^{-\alpha} k(t)|u|^{p} d t
$$


By [15], we get

$$
\int_{\mathbf{H}^{N}}\left|\nabla_{\mathbf{H}^{N}} u\right|^{2} d V_{\mathbf{H}^{N}}=w_{N-1} \int_{0}^{\infty} k(t)\left|u^{\prime}\right|^{2} d t
$$

$$
\left\|(\sinh t)^{\frac{N-1}{2}} u(t)\right\|_{H^{s}(1, \infty)} \leq C\|u\|_{H_{r}^{s}\left(\mathbf{H}^{N}\right)} .
$$

Thus, we can easily deduce this lemma by $\forall p \in\left(2, \frac{2(N-\alpha)}{N-2 s}\right)$,

$$
\begin{aligned}
& \|u\|_{L_{\alpha}^{p}\left(\mathbf{H}^{N}\right)} \leq C\|u\|_{H^{s}\left(\mathbf{H}^{N}\right)}, \quad \forall u \in H_{r}^{s}\left(\mathbf{H}^{N}\right), \\
& \left\|(\sinh t)^{\frac{N-1}{2}} u(t)\right\|_{L_{\alpha}^{p}(1, \infty)} \leq C\|u\|_{H_{r}^{s}\left(\mathbf{H}^{N}\right)} .
\end{aligned}
$$

Indeed, we have

$$
\begin{aligned}
\int_{R}^{\infty} t^{\alpha}(\sinh t)^{N-1}|u(t)|^{p} d t & \leq \frac{1}{\left(\frac{e^{R}+e^{-R}}{2}\right)^{(N-1)\left(\frac{p}{2}-1\right)}} \int_{R}^{\infty} t^{\alpha}(\sinh t)^{\frac{(N-1) p}{2}}|u(t)|^{p} d t \\
& \leq \frac{1}{\left(\frac{e^{R}+e^{-R}}{2}\right)^{(N-1)\left(\frac{p}{2}-1\right)}}\|u\|_{H^{s}\left(\mathbf{H}^{N}\right) .}
\end{aligned}
$$

It implies that if $R$ is large enough,

$$
w_{N-1} \int_{R}^{\infty} t^{\alpha}(\sinh t)^{N-1}|u(t)|^{p} d t \leq \varepsilon, \quad \forall u \in H_{r}^{s}\left(\mathbf{H}^{N}\right) .
$$

Similar as Lemma 3.2, we may verify that

Lemma 3.3. Let $s>0$. The restriction to $H_{r}^{s}\left(\mathbf{H}^{N}\right)$ of the Sobolev imbedding of $H^{s}\left(\mathbf{H}^{N}\right)$ into $M_{\beta}^{q}\left(\mathbf{H}^{N}\right)$ is continuous if $2 \leq q \leq \frac{2(N+\beta)}{N-2 s}$, and it is compact if $2<q<\frac{2(N+\beta)}{N-2 s}$.

Now, let $s, t>0$ with $s+t=2$, we define the Hilbert space $E=H_{r}^{s}\left(\mathbf{H}^{N}\right) \times$ $H_{r}^{t}\left(\mathbf{H}^{N}\right)$ and the bilinear form $B: E \times E \longrightarrow \mathbf{R}$ is defined by

$$
B[(u, v),(\varphi, \psi)]=\int_{\mathbf{H}^{N}} A^{s} u A^{t} \psi+A^{s} \varphi A^{t} v,
$$

and the corresponding quadratic form associated with the bilinear form $B$ is

$$
Q(z)=\int_{\mathbf{H}^{N}} A^{s} u A^{t} v, \quad(u, v) \in E .
$$

As in [11], we have $E=E^{-} \oplus E^{+}$and $B\left[z^{+}, z^{-}\right]=0$ for $z^{+} \in E^{+}, z^{-} \in E^{-}$, where

$$
E^{-}=\left\{\left(u,-A^{-t} A^{s} u\right): u \in H_{r}^{s}\left(\mathbf{H}^{N}\right)\right\}, \quad E^{+}=\left\{\left(u, A^{-t} A^{s} u\right): u \in H_{r}^{s}\left(\mathbf{H}^{N}\right)\right\} .
$$

We also have $Q(z)=\frac{1}{2} B[z, z]$ and $\frac{1}{2}\|z\|_{E}^{2}=Q\left(z^{+}\right)-Q\left(z^{-}\right)$, where $z=(u, v) \in E$ and $z=z^{+}+z^{-}, z^{+} \in E^{+}, z^{-} \in E^{-}$.

Now, we consider the existence of solutions for problem (1.1). We choose $s, t>0$, and $s+t=2$ such that

$$
2<p+1<\frac{2 N}{N-2 t}, \quad 2<q+1<\frac{2 N}{N-2 s} .
$$

By the assumptions in Theorem 1.1, we know that such an $s$ exists.

We consider the functional $I: E \longrightarrow \mathbf{R}$, defined by $I(z)=$

$$
\int_{\mathbf{H}^{N}} A^{s} u A^{t} v d V_{\mathbf{H}^{N}}-\frac{1}{p+1} \int_{\mathbf{H}^{N}} \frac{1}{(d(x))^{\alpha}}|v|^{p+1} d V_{\mathbf{H}^{N}}-\frac{1}{q+1} \int_{\mathbf{H}^{N}} \frac{1}{(d(x))^{\beta}}|u|^{q+1} d V_{\mathbf{H}^{N}}
$$


for $z=(u, v) \in E . I$ is a $C^{1}$ functional and

$$
\left\langle I^{\prime}(z), \eta\right\rangle_{E}=\int_{\mathbf{H}^{N}} A^{s} u A^{t} \psi+A^{s} \varphi A^{t} v-\int_{\mathbf{H}^{N}} \frac{1}{(d(x))^{\alpha}}|v|^{p-1} v \psi-\int_{\mathbf{H}^{N}} \frac{1}{(d(x))^{\beta}}|u|^{q-1} u \varphi
$$

for $z=(u, v) \in E$ and $\eta=(\varphi, \psi) \in E$. So the critical points of $I$ satisfy the equations

and

$$
\int_{\mathbf{H}^{N}} A^{s} u A^{t} \psi-\int_{\mathbf{H}^{N}} \frac{1}{(d(x))^{\alpha}}|v|^{p-1} v \psi=0 \quad \text { for all } \psi \in H_{r}^{t}\left(\mathbf{H}^{N}\right),
$$

$$
\int_{\mathbf{H}^{N}} A^{s} \varphi A^{t} v-\int_{\mathbf{H}^{N}} \frac{1}{(d(x))^{\alpha}}|u|^{q-1} u \varphi=0 \quad \text { for all } \varphi \in H_{r}^{s}\left(\mathbf{H}^{N}\right) .
$$

Let $\phi_{n}$ be the eigenfunction of $-\Delta_{\mathbf{H}^{N}}$ in $H_{r}^{1}\left(\mathbf{H}^{N}\right)$. Let

$$
E_{n}=\operatorname{span}\left\{\phi_{1}, \phi_{2}, \cdots, \phi_{n}\right\} \times \operatorname{span}\left\{\phi_{1}, \phi_{2}, \cdots, \phi_{n}\right\} .
$$

It is easy to see that $\overline{\bigcup_{n=1}^{\infty} E_{n}}=E$.

As $A^{s}: H_{r}^{s}\left(\mathbf{H}^{N}\right) \rightarrow L_{r}^{2}\left(\mathbf{H}^{N}\right)$ and $A^{t}: H_{r}^{t}\left(\mathbf{H}^{N}\right) \rightarrow L_{r}^{2}\left(\mathbf{H}^{N}\right)$ are isomorphisms, we see that $\left\{\hat{\phi}_{j}\right\}, j=1,2, \cdots$, where $\hat{\phi}_{j}=A^{-t} A^{s} \phi_{j}$ is a complete orthogonal system in $H_{r}^{t}\left(\mathbf{H}^{N}\right)$.

Now we define the splitting of $E_{n}$. For $K \in \mathbf{N}$ and for $n \geq K$, let

$$
X_{n}=\left(E_{1}^{-} \oplus \cdots \oplus E_{n}^{-}\right) \oplus\left(E_{1}^{+} \oplus \cdots \oplus E_{k-1}^{+}\right) \quad \text { and } \quad Y_{n}=\left(E_{k}^{+} \oplus \cdots \oplus E_{n}^{+}\right)
$$

where $E_{j}^{+}=\operatorname{span}\left\{\left(\phi_{j}, \hat{\phi}_{j}\right)\right\}$ and $E_{j}^{-}=\operatorname{span}\left\{\left(\phi_{j},-\hat{\phi}_{j}\right)\right\}$. We have $E_{n}=X_{n} \oplus Y_{n}$.

We say that the functional $I$ satisfies the so-called $(P S)^{*}$ condition with respect to $E_{n}$, i.e. any sequence $\left\{z_{k}\right\}, z_{k} \in E_{n_{k}}$ with $n_{k} \rightarrow \infty$ as $k \rightarrow \infty$, satisfying $\left.I^{\prime}\right|_{E_{n_{k}}}\left(z_{k}\right) \rightarrow$ 0 and $I\left(z_{k}\right) \rightarrow c$ has a subsequence that converges in $E$.

The result in [8] now can be stated as follows.

Lemma 3.4. Suppose that $I \in C^{1}(E, \mathbf{R})$ be an even functional satisfying the $(P S)^{*}$ condition with respect to $E_{n}$. Assume that $T: E_{n} \rightarrow E_{n}$ for $n$ large. Let $\rho>0$ and $\sigma>0$ be such that $\sigma\left\|T y_{1}\right\|_{E}>\rho$. Assume that there are constants $\alpha \leq \beta$ such that

$$
\inf _{S \cap E_{n}} I \geq \alpha, \quad \sup _{T\left(\partial D \cap E_{n}\right)} I<\alpha, \quad \text { and } \sup _{T\left(D \cap E_{n}\right)} I \leq \alpha
$$

for $n$ large. Then $I$ has a critical value $c \in[\alpha, \beta]$.

Lemma 3.5. The functional $I$ satisfies the $(P S)^{*}$ condition with respect to $E_{n}$.

Proof. Let $\left(z_{k}\right)_{k \geq 1}=\left(u_{k}, v_{k}\right)_{k \geq 1} \subset E_{n_{k}}$ be a sequence such that

$$
I\left(z_{k}\right) \rightarrow c,\left.\quad I^{\prime}\right|_{E_{n_{k}}}\left(z_{k}\right) \rightarrow 0 .
$$

We need only to show that $\left\|z_{n}\right\|=\left\|\left(u_{n}, v_{n}\right)\right\|$ is bounded uniformly. Indeed, if $\left\|z_{n}\right\|$ is uniformly bounded, by using Lemma 3.2 , we see that $u_{n}$ and $v_{n}$ converge strongly up to a subsequence in $L^{p+1}$ and $L^{q+1}$ respectively, the conclusion here doesn't follow in a standard way but can be proved as in [11]. Taking $\eta=z_{k}$, we have

$$
\begin{aligned}
c+1+\varepsilon_{k}\left\|z_{n}\right\| \geq & I\left(z_{k}\right)-\frac{1}{2}\left\langle I^{\prime}\left(z_{k}\right), z_{k}\right\rangle \\
= & \left(\frac{1}{2}-\frac{1}{p+1}\right) \int_{\mathbf{H}^{N}} \frac{1}{(d(x))^{\alpha}}\left|v_{k}\right|^{p+1} d V_{\mathbf{H}^{N}} \\
& +\left(\frac{1}{2}-\frac{1}{q+1}\right) \int_{\mathbf{H}^{N}} \frac{1}{(d(x))^{\beta}}\left|u_{k}\right|^{q+1} d V_{\mathbf{H}^{N}} .
\end{aligned}
$$


Now, we estimate $\left\|u_{k}\right\|_{H_{r}^{s}}$ and $\left\|v_{k}\right\|_{H_{r}^{t}}$. From (3.3), we have

$$
\begin{aligned}
& \left|\int_{\mathbf{H}^{N}} A^{s} \varphi A^{t} v_{n}\right| \leq\left.\left|\int_{\mathbf{H}^{N}} \frac{1}{(d(x))^{\beta}}\right| u_{k}\right|^{q-1} u_{k} \varphi d V_{\mathbf{H}^{N}} \mid+\varepsilon_{k}\|\varphi\|_{H_{r}^{s}} \\
& \leq\left(\int_{\mathbf{H}^{N}} \frac{1}{(d(x))^{\beta}}\left|u_{k}\right|^{q+1} d V_{\mathbf{H}^{N}}\right)^{\frac{q}{q+1}}\left(\int_{\mathbf{H}^{N}} \frac{1}{(d(x))^{\beta}}|\varphi|^{q+1} d V_{\mathbf{H}^{N}}\right)^{\frac{1}{q+1}}+\varepsilon_{k}\|\varphi\|_{H_{r}^{s}} \\
& \leq\left[\left(\int_{\mathbf{H}^{N}} \frac{1}{(d(x))^{\beta}}\left|u_{k}\right|^{q+1} d V_{\mathbf{H}^{N}}\right)^{\frac{q}{q+1}}+\varepsilon_{n}\right]\|\varphi\|_{H_{r}^{s}} \text { for all } \varphi \in E_{n_{k}}^{s}
\end{aligned}
$$

which implies that

$$
\left\|v_{n}\right\|_{H_{r}^{t}} \leq\left(\int_{\mathbf{H}^{N}} \frac{1}{(d(x))^{\beta}}\left|u_{n}\right|^{q+1} d V_{\mathbf{H}^{N}}\right)^{\frac{q}{q+1}}+\varepsilon_{n} .
$$

Similarly, we prove that

$$
\left\|u_{n}\right\|_{H_{r}^{s}} \leq\left(\int_{\mathbf{H}^{N}} \frac{1}{(d(x))^{\alpha}}\left|v_{n}\right|^{p+1} d V_{\mathbf{H}^{N}}\right)^{\frac{p}{p+1}}+\varepsilon_{n} .
$$

Thus we conclude that

$$
\begin{aligned}
& \left\|u_{n}\right\|_{H_{r}^{s}}+\left\|v_{n}\right\|_{H_{r}^{t}} \\
& \leq\left(\int_{\mathbf{H}^{N}} \frac{1}{(d(x))^{\beta}}\left|v_{n}\right|^{p+1} d V_{\mathbf{H}^{N}}\right)^{\frac{p}{p+1}}+\left(\int_{\mathbf{H}^{N}} \frac{1}{(d(x))^{\alpha}}\left|u_{n}\right|^{q+1} d V_{\mathbf{H}^{N}}\right)^{\frac{q}{q+1}}+2 \varepsilon_{n} .
\end{aligned}
$$

Using this estimate in (3.3), we get

$$
\left\|u_{n}\right\|_{L_{\beta}^{q+1}}^{q+1}+\left\|v_{n}\right\|_{L_{\alpha}^{p+1}}^{p+1} \leq C\left(\left\|u_{n}\right\|_{L_{\beta}^{q+1}}^{q}+\left\|v_{n}\right\|_{L_{\alpha}^{p+1}}^{p}\right)+C .
$$

Hence, both $\left\|u_{n}\right\|_{L_{\beta}^{q+1}},\left\|v_{n}\right\|_{L_{\alpha}^{p+1}}$ are bounded, and consequently $\left\|u_{n}\right\|_{H_{r}^{s}},\left\|v_{n}\right\|_{H_{r}^{t}}$ are also bounded in view of (3.4).

Proof of Theorem 1.1. Thanks to Lemma 3.5, we know that $I$ satisfies the $(P S)^{*}$ condition and hence using the abstract critical point result [8], we obtain that the functional $I$ has a critical value $c_{k} \in\left[\alpha_{k}, \beta_{k}\right]$. Since $\alpha_{k} \rightarrow \infty$, we get infinitely many critical values of $I$. Therefore we have infinitely many solution of (1.11).

Proofs of Theorems 1.2 and 1.3. The proofs of Theorems 1.2 and 1.3 are similar to that of Theorem 1.1. We only give the main ideas of the proof. We may choose $s+t=2$ under assumptions of Theorem 1.2, so that the inclusions $i_{1}: E^{s} \times E^{t} \rightarrow$ $M_{\alpha}^{p+1} \times M_{\beta}^{q+1}$ is compact. Using Lemma 3.5 , we may verify $(P S)^{*}$ condition. The rest part are similar to the proof of Theorem 1.1, we omit the details.

Acknowledgements. This work is supported by the scholarship from China Scholarship Council (CSC) under the Grant CSC (No: 201308430355), the National Natural Sciences Foundations of China(No:11201140), and Hunan Provincial Natural Science Foundation of China (No:14JJ6005).

\section{References}

[1] Azorero, J. P. G., and I. P. Alonso: Hardy inequalities and some critical elliptic and parabolic problems. - J. Differential Equations 144, 1998, 441-476.

[2] Badiale, M., and E. Serra: Multiplicity results for the supercritical Hénon equation. - Adv. Nonlinear Stud. 4, 2004,453-467. 
[3] Bandle, C., A. Brillard, and M. Flucher: Green's function, harmonic transplantion and best Sobolev constant in spaces of constant curvature. - Trans. Amer. Math. Soc. 350, 1998, $1103-1128$.

[4] Bhakta, M., and K. Sandeep: Poincaré Sobolev equations in the hyperbolic space. - Calc. Var. Partial Differential Equations 44, 2012, 247-269.

[5] Bonforte, M., F. Gazzola, G. Grillo, and J. L. VÀzquez: Classification of radial solutions to the Emden-Fowler equation on the hyperbolic space. - Calc. Var. Partial Differential Equations 46, 2013, 375-401.

[6] Caffarelli, L., R. Kohn, and L. Nirenberg: First order interpolation inequalities with weights. - Compositio Math. 53, 1984, 259-275.

[7] Ferrero, A., and F. Gazzola: Existence of solutions for singular critical growth semilinear elliptic equations. - Differ. Equ. 177, 2001, 494-522.

[8] Felmer, P., and Z.-Q. WANG: Multiplicity for symmetric indefinite functionals: application to Hamiltonian and elliptic systems. - Topol. Methods Nonlinear Anal. 12, 1998, 207-226.

[9] De Figueiredo, D. G., and P. L. Felmer: On superquadratic elliptic systems. - Trans. Amer. Math. Soc. 343, 1994, 99-116.

[10] De Figueiredo, D. G., I. Peral, and J. D. Rossi: The critical hyperbola for a Hamiltonian elliptic system with weights. - Ann. Mat. Pura Appl. 187, 2008, 531-545.

[11] De Figueiredo, D. G., and J. YAng: Decay, symmetry and existence of solutions of semilinear elliptic systems. - Nonlinear Anal. 33, 1998, 211-234.

[12] GidAs, B., and J. SpRUCK: Global and local behavior of positive solutions of nonlinear elliptic equations. - Comm. Partial Differential Equations 17, 1992, 923-940.

[13] Ghoussoub, M., and C. Yuan: Multiple solutions for quasilinear PDEs involving the critical Sobolev and Hardy exponents. - Trans. Amer. Math. Soc. 352, 2000, 5703-5743.

[14] He, H.: The existence of solutions for Hénon equation in hyperbolic space. - Proc. Japan Acad. $89,2013,24-28$.

[15] He, H.: Symmetry and existence of solutions of semilinear elliptic systems in hyperbolic space. - Glasgow Math. J. (to appear).

[16] Hulshof, J., and R. VAn DER Vorst: Differential systems with strongly indefinite variational structure. - J. Funct. Anal. 114, 1993, 32-58.

[17] JIE, Q: A priori esimates for positive solutions of semilinear elliptic systems. - J. Partial Differential Equations 1, 1988, 525-598.

[18] Liu, F., and J. YAng: Nontrivial solutions of Hardy-Hénon type elliptic systems. - Acta Math. Sci. 27, 2007, 673-688.

[19] Mancini, G., and K. Sandeep: On a semilinear elliptic equaition in $\mathbf{H}^{N}$. - Ann. Sc. Norm. Super. Pisa Cl. Sci. 7, 2008, 635-671.

[20] Ni, W. M.: A nonlinear Dirichlet problem on the unit ball and its applications. - Indiana Univ. Math. J. 31, 1982, 801-807.

[21] Pistoia, A., and E. Serra: Multi-peak solutions for the Hénon equation with slightly subcritical growth. - Math. Z. 256, 2007, 75-97.

[22] Serra, E.: Nonradial positive solutions for the Hénon equation with critical growth. - Calc. Var. Partial Differential Equations 23, 2005, 301-326.

[23] Smets, D., J. B. Su, and M. Willem: Non-radial ground states for the Hénon equation. Comm. Contemp. Math. 4, 2002, 467-480.

[24] Smets, D., and M. Willem: Partial symmetry and asymptotic behavior for some elliptic variational problem. - Calc. Var. Partial Differential Equations 18, 2005, 57-75. 
[25] Souto, M. A.: Sobre a existencia de solucoes positivas para sistemas cooperativos nao lineares. - PhD thesis, Unicamp, 1992.

[26] Stapelkamp, S.: The Brezis-Nirenberg problem on $\mathbf{H}^{n}$ : Existence and uniqueness of solutions. - In: Elliptic and Parabolic Problems (Rolduc and Gaeta 2001), World Scientific, Singapore, 2002, 283-290.

[27] Stapelkamp, S.: Das Brezis-Nirenberg problem on $\mathbf{H}^{n}$. - Dissertation, Universität Basel, 2003.

[28] Triebel, H.: Theory of function spaces II. - Monographs Math. 84, Birkhäuser, 1992.

[29] VAN DER Vorst, R.: Variational identities and applications to differential systems. - Arch. Ration. Mech. Anal. 116, 1991, 375-398.

[30] Wolf, J.: Spaces of constant curvature. - McGraw-Hill, New York, 1967.

Received 4 December $2014 \bullet$ Accepted 17 April 2015 